\title{
Pionic hydrogen and deuterium
}

\author{
D. Gotta ${ }^{1 \star}$ and L.M. Simons ${ }^{2}$ \\ 1 Institut für Kernphysik, Forschungszentrum Jülich GmbH, D-52425 Jülich, Germany \\ 2 Paul Scherrer Institut, CH-5232 Villigen, Switzerland \\ *d.gotta@fz-juelich.de \\ February 4, 2021
}

Review of Particle Physics at PSI

doi:10.21468/SciPostPhysProc.2

\section{Abstract}

\begin{abstract}
The measurement of strong-interaction shift and broadening in pionic hydrogen and deuterium yields pion-nucleon scattering lengths as well as the threshold pion-production strength on isoscalar $N N$ pairs. Results from recent high-resolution experiments at PSI using crystal spectrometers allow important comparisons with the outcome of the modern low-energy description of QCD within the framework of effective field theories.
\end{abstract}

\subsection{Introduction}

The last decades have seen a successful theoretical description of strong-interaction phenomena at threshold within effective field-theory (EFT) approaches: the chiral symmetry of the QCD Lagrangian allows the derivation of so-called low-energy theorems. In reality, the chiral symmetry is explicitly broken because of the finite masses of the light quarks $u, d$, and $s$. This leads, e.g. to a finite pion mass which is, however, still small compared to the hadronic scale given by the nucleon mass. The deviations from low-energy theorems reveal the amount of symmetry breaking.

Chiral Perturbation Theory ( $\chi$ PT) offers a systematic way of quantifying these symmetrybreaking effects. A chiral expansion, ordered by the powers of (small) momenta, the quarkmass differences, and the fine structure constant $\alpha$, includes strong isospin-breaking effects resulting from the quark-mass differences and those of electromagnetic origin on the same footing. The unknown structure of QCD at short distances is parametrized by so-called lowenergy constants (LECs), which must be taken from experiment as long as results from latticeQCD calculations are not available.

Pions, being composite particles of the lightest quarks $u$ and $d$, and their interactions, play a prominent role. Hence, $\pi N \rightarrow \pi N$ reactions and the corresponding scattering lengths are of fundamental interest for the understanding of low-energy QCD phenomena. In the limit of isospin conservation, all $\pi N \rightarrow \pi N$ reactions are completely determined by only two independent real numbers, the scattering lengths corresponding to the isospin combinations $I=1 / 2$ and $3 / 2$ of the $\pi N$ system. Therefore, quantitative tests of isospin-breaking effects, predicted to be of the order of a few per cent by advanced $\chi$ PT calculations, are of great importance.

The corresponding precision for the experimental approach is achieved by means of highresolution X-ray spectroscopy of pionic hydrogen and deuterium. Considering the energy regime of such atomic systems, the measurement of the strong-interaction effects constitutes a scattering experiment at threshold. 
Concepts and recent theoretical efforts on low-energy $\pi N$ scattering and pionic hydrogen are reviewed in $[1,2]$. Properties of exotic atoms and experimental methods are outlined in [3].

\subsection{Strong-interaction effects}

Exotic atoms provide an ideal laboratory for the extraction of scattering lengths from experiment, because problems due to normalization and extrapolation to threshold inherent to scattering experiments are absent. Such atoms are formed when negatively charged particles, such as pions, are captured in high-lying atomic levels of the Coulomb potential of a nucleus: a de-excitation cascade subsequently starts. The strong interaction gives rise to a change of the total energy of the particle-nucleus system $\Delta E$ and to its lifetime observed as an energy shift $\epsilon$ and a broadening $\Gamma$ of lower-lying atomic levels, where the overlap of the atomic bound-state wave function with the nucleus of mass number $A$ becomes significant. For atomic states with principle quantum number $n$ and angular momentum $\ell=0, n s$, the complex energy shift is directly related to the complex scattering length $a_{\pi A}[4]$

$$
\Delta E_{n s}^{\pi \mathrm{A}}-i \frac{\Gamma_{n s}^{\pi \mathrm{A}}}{2}=-\frac{2 \alpha^{3} \mu_{\mathrm{A}}^{2} c^{4}}{\hbar c} \cdot \frac{1}{n^{3}} \cdot a_{\pi \mathrm{A}}+\ldots,
$$

where $\alpha$ is the fine structure constant and $\mu_{A}$ is the reduced mass of the particle-nucleus system. The ellipses stand for higher order corrections $[1,2]$. In this paper, the sign convention for atomic level shifts $\epsilon$ is the change of the X-ray transition energy, i.e. $\epsilon \equiv-\Delta E$.

In the case of pionic hydrogen, only the ground-state effects are detectable by high-resolution X-ray spectroscopy. The two independent scattering lengths may be described by isoscalar and isovector scattering lengths $a^{+}$and $a^{-}$for the elastic channels:

$$
a^{ \pm} \equiv\left(a_{\pi^{-} p \rightarrow \pi^{-} p} \pm a_{\pi^{+} p \rightarrow \pi^{+} p}\right) .
$$

$a^{+}$and $a^{-}$are given in term of the isospin combinations $I=1 / 2$ and $\mathrm{I}=3 / 2$ by:

$$
\begin{aligned}
& a^{+}=\frac{1}{3}\left(a_{1 / 2}+2 a_{3 / 2}\right) \text { and } \\
& a^{-}=\frac{1}{3}\left(a_{1 / 2}-a_{3 / 2}\right) .
\end{aligned}
$$

In leading order, pionic hydrogen and deuterium give access to the scattering lengths of the elastic reactions $\pi^{-} p \rightarrow \pi^{-} p$ and $\pi^{-} n \rightarrow \pi^{-} n$ and to the charge exchange channel $\pi^{-} p \rightarrow \pi^{0} n$ after correcting for the radiative capture contribution $\pi^{-} p \rightarrow \gamma n$. As seen from (14.5) - (14.7), three experimental quantities are available for the two independent scattering lengths: the $1 s$ level strong-interaction shifts in pionic hydrogen and deuterium $\epsilon_{1 s}^{\pi \mathrm{H}}$ and $\epsilon_{1 s}^{\pi \mathrm{D}}$ and broadening in pionic hydrogen, $\Gamma_{1 s}^{\pi \mathrm{H}}$. Hence, such measurements constitute a decisive constraint both on the experimental and theoretical approaches.

$$
\begin{aligned}
\epsilon_{1 s}^{\pi \mathrm{H}} \propto \operatorname{Re} a_{\pi^{-} p} \propto a_{\pi^{-} p \rightarrow \pi^{-} p} & =a^{+}+a^{-}+\ldots \\
\Gamma_{1 s}^{\pi \mathrm{H}} \propto \mathfrak{S} a_{\pi^{-} p} \propto\left(a_{\pi^{-} p \rightarrow \pi^{0} n}\right)^{2} & =\left(a^{-}\right)^{2}+\ldots \\
\epsilon_{1 s}^{\pi \mathrm{D}} \propto \operatorname{Re} a_{\pi^{-} d} \propto a_{\pi^{-} p \rightarrow \pi^{-} p}+a_{\pi^{-} n \rightarrow \pi^{-} n} & =2 \cdot a^{+}+\ldots .
\end{aligned}
$$

The ellipses indicate the corrections needed to derive the QCD quantities $a^{+}$and $a^{-}$from the measurable quantities $a_{\pi N \rightarrow \pi N}$. These corrections are given by recent $\chi$ PT calculations $[1,2]$. For the pionic deuterium case, in addition substantial multi-body corrections are necessary which, however, are well under control [5]. The check of consistency of these results is an essential outcome of the experimental and theoretical efforts of the last decades. 
The imaginary part $\operatorname{Im} a_{\pi \mathrm{D}}$, which gives the leading contribution to the hadronic broadening $\Gamma_{1 s}^{\pi \mathrm{D}}$ in pionic deuterium, measures the transition strength $\alpha$ of $s$-wave pions on an isoscalar nucleon-nucleon pair $\pi N N \leftrightarrow N N$ and is an independent quantity not related to the scattering lengths $a^{+}$and $a^{-}[6,7]$.

$$
\Gamma_{1 s}^{\pi \mathrm{D}} \propto \mathfrak{I} a_{\pi^{-} d} \propto \sigma_{\pi^{+} d \rightarrow p p}^{\text {threshold }} \propto \alpha
$$

\subsection{Experimental approach}

The possibility of performing high-statistics experiments of exotic hydrogen even in dilute targets with high-resolution devices like crystal spectrometers became possible by using the cyclotron trap (Section ??). Figure 14.1 shows the set-up of cyclotron trap and crystal spectrometer for the studies described here [7-10]. With a massive specially tailored concrete shielding an improvement in the beam-induced background of up to a factor of 50 is achieved compared to previous experiments.

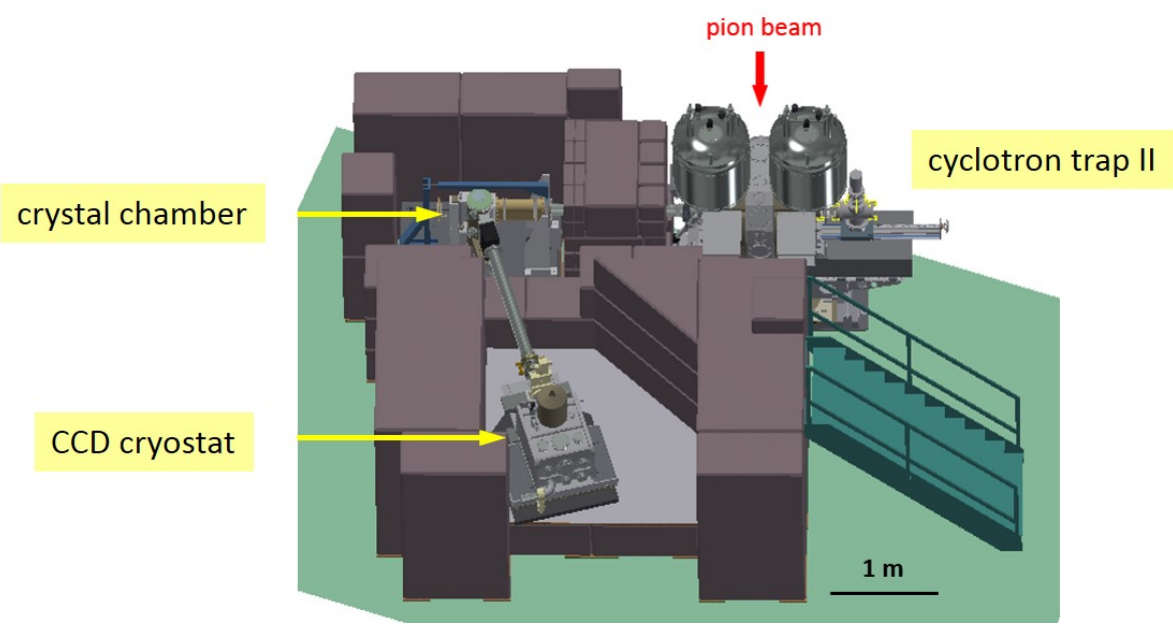

Figure 14.1: Set-up in the $\pi \mathrm{E} 5$ area at PSI. The Bragg crystal is mounted inside a vacuum chamber and connected to the cyclotron trap and the CCD X-ray detector by a vacuum system to minimize absorption losses. The roof of the concrete cave is not shown.

The crystal spectrometer was set up in Johann configuration which allows the measurement of an energy interval corresponding to the extension of the X-ray source in the direction of dispersion [11]. Thin polished slabs of silicon and quartz were used as Bragg crystals. They are spherically bent with radii of about $R_{c}=3 \mathrm{~m}$ when attached to glass lenses of optical quality by adhesive forces only (Figure 14.2). Resolutions of $270-460 \mathrm{meV}$ were achieved for the X-rays in the energy range of $2.2-3.1 \mathrm{keV}$ which is very close to the theoretical limit achievable for the particular crystal and reflection.

The detector extension in the direction of dispersion has to be matched to the source size to utilize the capabilities of the Johann set-up. The detector is realized as a $2 \times 3$ array of Charge-Coupled Device (CCD) of total area of $48 \times 72 \mathrm{~mm}^{2}$ (Figure 14.2) and located at the distance $R_{C} \cdot \sin \Theta_{B}$ given by the focussing condition where $\Theta_{B}$ is the Bragg angle. The diffracted $\mathrm{X}$-rays create a cone-like hit pattern in the detection plane which, after curvature correction and projection to the direction of dispersion, directly yields an energy spectrum (Figure 14.3). The necessary two-dimensional position resolution is provided by the $40 \mu \mathrm{m} \times 40 \mu \mathrm{m}$ pixel structure of the CCDs. 

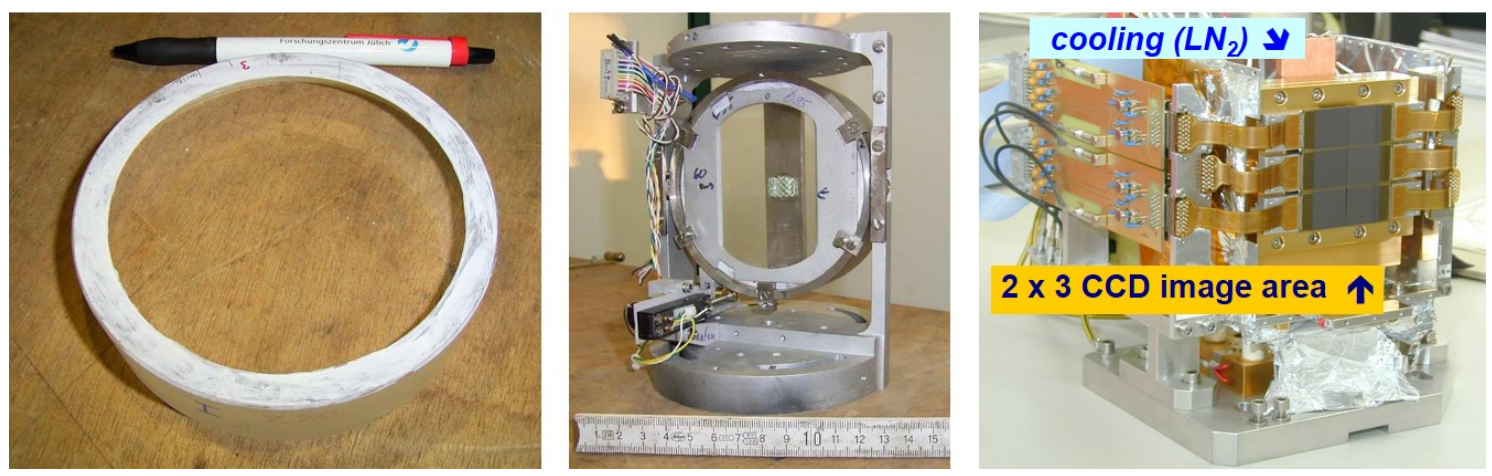

Figure 14.2: Left: quartz disk attached adhesively to a concave glass lens, middle: crystal mounting in an adjustable support frame with an aperture to limit the so-called Johann defocussing, right: focal-plane X-ray detector removed from the cryostat [12].

Energy determination in Johann-type set-ups requires a calibration line ideally at the same Bragg angle as the X-ray line of interest. In this experiment, the energy of the pionic oxygen line $(6-5)$ is very close to the one of pionic hydrogen $(3-1)$. The precise knowledge of the charged pion mass then allows the calibration of pionic-atom transitions among themselves [13] (Section 10 [14]).

The understanding of collisional processes during the lifetime of pionic hydrogen plays a key role for a precision determination of the strong-interaction effects. For the $\pi \mathrm{H}$ system, $\mathrm{X}$-ray transition energies are of the order of $3 \mathrm{keV}$ while hadronic shifts are of the order of a few $\mathrm{eV}$ and the broadening around $1 \mathrm{eV}$. Therefore, a thorough study of possible collisioninduced energy shifts and broadening has been performed. Such a study essentially means the measurement of various transitions at various target densities as well as a comparison with muonic hydrogen. The hydrogen density was adjusted in the cryogenic target by temperature variation in order to allow the use of thin windows.

Energy shifts, which would spoil the result for $\epsilon_{1 s}$, may appear if after molecular formation $\pi \mathrm{H}+\mathrm{H}_{2} \rightarrow[(p p \pi) p]$ ee X-ray emission from molecular states occurs. As the formation rate scales with collision probability, a density-dependent X-ray energy would demonstrate its appearance. No such effect was observed for either hydrogen and deuterium [7-9].

The main obstacle to a precision determination of the hadronic broadening $\Gamma_{1 s}$ is Doppler broadening due to Coulomb de-excitation. During these non-radiative transitions, the energy of the de-excitation step $\left(n-n^{\prime}\right)$ is transferred into kinetic energy of the collision partners. The competition of acceleration by Coulomb de-excitation and deceleration by elastic and inelastic collisions leads to a complex kinetic energy distribution at the time of X-ray emission. Hence, the measured line shape is a convolution of spectrometer response, Doppler broadening, and the Lorentzian representing the hadronic broadening. For that reason, a measurement of the twin system muonic hydrogen was performed, where the absence of the strong interaction allows the possibility of directly studying the effect of Doppler broadening.

Consequently, the ultimate knowledge of the spectrometer response is of great importance. Here, the cyclotron trap offers another unique possibility when extended to operate as ECR source (Section 13 [15]). The ECR source yields narrow X-rays at high rates from helium-like low $Z$ elements like sulphur, chlorine, and argon which almost coincide in energy with the pionic hydrogen and deuterium X-ray transitions. 


\subsection{Results}

Spectra of the $(3 p-1 s)$ transitions are shown in Figure 14.3. Above the oxygen freeze-out temperature the simultaneous measurement of the $\pi \mathrm{O}$ calibration line and $\pi \mathrm{H}$ line is feasible by means of a small $\mathrm{O}_{2}$ admixture to the $\mathrm{H}_{2}$ gas. For lower temperature, hydrogen and oxygen measurements were performed alternately.
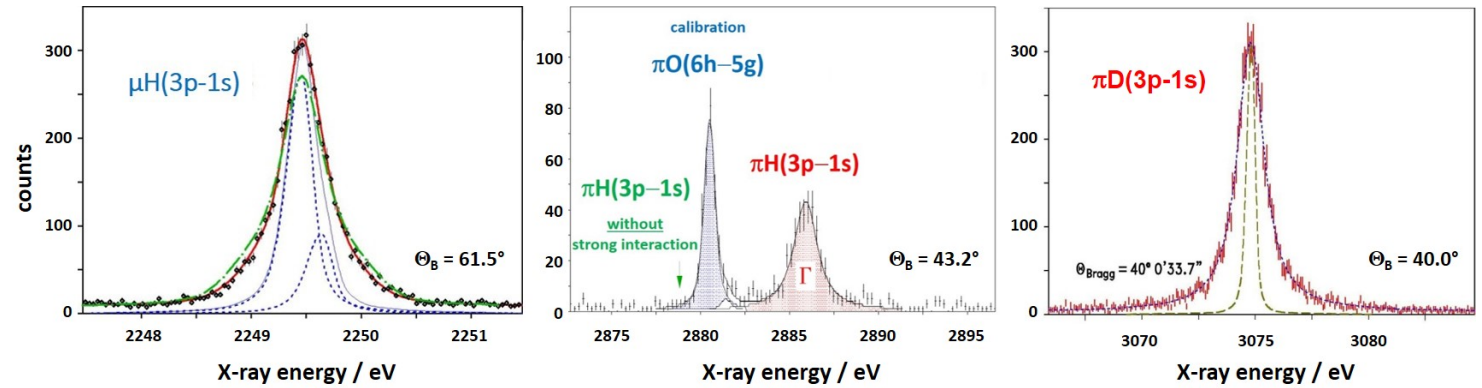

Figure 14.3: Spectra of the $(3 p-1 s)$ transitions in muonic [8] and pionic hydrogen [9] and pionic deuterium [7]. The narrow structures displayed inside the $\mu \mathrm{H}$ and $\pi \mathrm{D}$ lines demonstrate the resolution of the spectrometer equipped with a Si 111 crystal as measured by using an ECR source [16].

The muonic hydrogen spectrum shows the importance of the Doppler-induced broadening (Figure 14.3). A satisfactory description requires that about $2 / 3$ of the $\mu \mathrm{H}$ atoms have kinetic energies below a few eV contributing only to a negligible amount to the broadening. The remaining $1 / 3$ can be attributed to energies around 24 and $56 \mathrm{eV}$, which corresponds to the Coulomb de-excitation transitions $(5-4)$ and $(4-3)$. Within the uncertainties of such an analysis, there is good agreement with cascade theory [8].

In pionic hydrogen, again a large fraction, about $60-80 \%$, of the $\pi \mathrm{H}$ atoms have kinetic energies below a few eV. The appearance of higher energies is needed to describe the line shape. However, because of the hadronic broadening an assignment to particular Coulomb de-excitation transitions is impossible [10].

It is worth mentioning that no Doppler contribution could be identified in pionic deuterium within the experimental uncertainties [7]. A theoretical explanation for such behavior is provided by cascade theory [17].

The strong-interaction effects, summarized in Table 14.1, represent the weighted average over the various transitions measured and target densities.

$$
\begin{array}{|cccc|}
\hline \epsilon_{1 s}^{\pi \mathrm{H}} & \Gamma_{1 s}^{\pi \mathrm{H}} & \epsilon_{1 s}^{\pi \mathrm{D}} & \Gamma_{1 s}^{\pi \mathrm{D}} \\
(7085.8 \pm 9.6)[9] & 856 \pm 27[10] & -2356 \pm 31[7] & 1171_{-49}^{+23}[7] \\
\hline
\end{array}
$$

Table 14.1: Measured strong-interaction effects in pionic hydrogen and deuterium (in $\mathrm{meV}$ ).

\subsection{Summary}

The three constraints on the two independent isoscalar and isovector $\pi N$ scattering lengths are shown in Figure 14.4. Because of the poor knowledge of LECs, the use of a modified isoscalar scattering length $\tilde{a}^{+}$is more convenient in the constraint analyses. The most recent 
$\chi$ PT calculation gives $\tilde{a}^{+}-a^{+}=(-6.1 \pm 2.5) \cdot 10^{-3} m_{\pi}^{-1}$ [2]. It is important to note that good overlap is achieved, although substantial chiral corrections have to be applied [2].

The precise result for the pion-production strength $\alpha$ demonstrates the advantage of exotic atoms, namely that the strong-interaction effects are determined without normalisation and extrapolation. In Figure 14.5, the pionic- deuterium results are marked as the shaded area, which is compared with pion-production experiments that typically specify statistical errors only. The only theoretical approach yielding a reliable uncertainty is due to a $\chi$ PT calculations which, however, suffers at present from the scarce precision of some LECs [6].

\begin{tabular}{|ccc|}
\hline$\tilde{a}^{+}$ & $a^{-}$ & $\alpha$ \\
$(1.7 \pm 0.8) \cdot 10^{-3} m_{\pi}^{-1}[10]$ & $(86.6 \pm 1.0) \cdot 10^{-3} m_{\pi}^{-1}[10]$ & $251_{-11}^{+5} \mathrm{mb}[7]$ \\
\hline
\end{tabular}

Table 14.2: Isoscalar and isovector scattering length $\tilde{a}^{+}$and $a^{-}$and threshold pionproduction strength as derived from the strong-interaction effects in pionic hydrogen and deuterium.

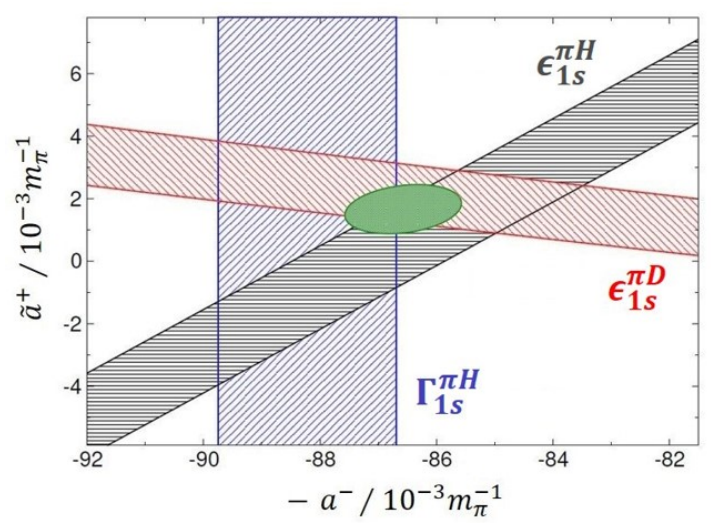

Figure 14.4: Constraints (bands) and combined result (ellispse) for the isoscalar and isovector $\pi N$ scattering lengths $\tilde{a}^{+}$and $a^{-}$as derived from $\epsilon_{1 s}^{\pi \mathrm{H}}$, $\epsilon_{1 s}^{\pi \mathrm{D}}$, and $\Gamma_{1 s}^{\pi \mathrm{H}}[10]$.

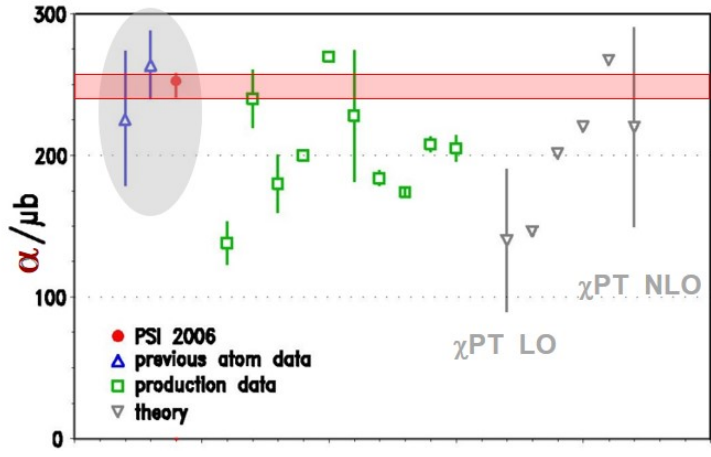

Figure 14.5: Comparison of results for pion-production strength $\alpha$ at threshold on isoscalar $N N$ pairs. The horizontal band represents the precision of the most recent result for $\Gamma_{1 s}^{\pi \mathrm{D}}[7]$.

Exotic-atom data yield values for the reaction scattering lengths $a_{\pi^{-} p \rightarrow \pi^{-} p}$ and $a_{\pi^{-} p \rightarrow \pi^{0} n}$ $[9,10]$. Applying the corrections provided by $\chi$ PT calculations, as well $a_{\pi^{+} p \rightarrow \pi^{+} p}$ as the isospinseparated scattering lengths $a_{1 / 2}$ and $a_{3 / 2}$ are attainable $[2,10]$. The results are in very good agreement with recent analyses of low-energy $\pi N$ scattering data [18].

In summary, recent $\pi H, \pi D$, and low-energy $\pi N$ scattering data are quantitatively very consistent when analysed within the framework of $\chi$ PT.

\section{References}

[1] J. Gasser, V. Lyubovitzkij and A. Rusetski, "Hadronic atoms in QCD + QED", Phys. Rep. 456, 167 (2008).

[2] M. Hoferichter et al., "Roy-steiner-equation analysis of pion-nucleon scattering", Phys. Rep. 625, 1 (2016). 
[3] D. Gotta, "Precision spectrosopy of light exotic atoms", Prog. Part. Nucl. Phys 52, 133 (2004).

[4] S. Deser et al., "Energy level disylacements in pi-mesonic atoms", Phys. Rev. 96, 774 (1954).

[5] V. Baru et al., "Precision calculation of threshold $\pi^{-} d$ scattering, $\pi N$ scattering lengths, and the GMO sum rule", Nucl. Phys. A 872, 69 (2011).

[6] V. Lensky et al., "Towards a field theoretic understanding of $\pi N N \rightarrow N N "$ ", Eur. Phys. J. A 27, 37 (2006).

[7] T. Strauch et al., "Pionic deuterium", Eur. Phys. J. A 47, 88 (2011).

[8] D. Covita et al., "Line shape analysis of the $K \beta$ transition in muonic hydrogen", Eur. Phys. J. D 72, 72 (2018).

[9] M. Hennebach et al., "Hadronic shift in pionic hydrogen", Eur. Phys. J. A 50, 190 (2014).

[10] A. Hirtl et al., "Redetermination of the strong-interaction width in pionic hydrogen", to be published in Eur. Phys. J. A (2021).

[11] D. Gotta and L. Simons, "Remarks on a Johann spectrometer for exotic-atom research and more", Spectrochim. Acta B 120, 9 (2016).

[12] N. Nelms et al., "A large area CCD X-ray detector for exotic atom spectroscopy", Nucl. Instr. Meth. A 484, 419 (2002).

[13] M. Trassinelli et al., "Measurement of the charged pion mass using X-ray spectroscopy of exotic atoms", Phys. Lett. B 759, 583 (2016).

[14] M. Daum and D. Gotta, The mass of the $\pi^{-}$, SciPost Phys. Proc. 2, ppp (2021), doi:10.21468/SciPostPhysProc.2.XXX.

[15] D. Gotta and L. Simons, Cyclotron trap, SciPost Phys. Proc. 2, ppp (2021), doi:10.21468/SciPostPhysProc.2.XXX.

[16] D. Anagnostopoulos et al., "On the characterisation of a Bragg spectrometer with $X$-rays from an ECR source", Nucl. Instr. and Meth. A 545, 217 (2005).

[17] V. Popov and V. Pomerantsev, "The isotopic effect in the scattering and kinetics of the atomic cascade of excited $\mu^{-} p$ and $\mu^{-} d$ atoms", Phys. Rev. A 95, 022506 (2017).

[18] J. Ruiz de Elvira et al., "Extracting the $\sigma$-term from low-energy pion-nucleon scattering", J. Phys. G 45, 024001 (2018). 